\title{
The importance of duodenal diverticula in the elderly
}

\author{
V. R. Pearce \\ B.Sc., M.B., M.R.C.P. \\ Chesterton Hospital, Cambridge
}

\begin{abstract}
Summary
All barium meal examinations performed, in patients aged $>65$ years, in one year in one Health District are reviewed. There were 39 cases of duodenal diverticula. One case of osteomalacia and folate deficiency was discovered and this patient had evidence of small bowel bacterial overgrowth. In the remaining cases showing evidence of nutritional deficiency, other factors were probably responsible. The evidence for an association between deficiencies and duodenal diverticula is discussed, and it is concluded that these structures are rarely responsible for nutritional deficiencies in the elderly.
\end{abstract}

\section{Introduction}

A number of isolated reports have shown that nutritional deficiencies may occur in the presence of duodenal diverticula (Brey et al., 1971; Gillespy, 1957; Goldstein, Cozzolina and Wirts, 1963). It has been suggested that in elderly subjects they are frequently associated with refractory anaemia and other deficiency states, and that the detection of these lesions at the time of barium meal examination should prompt a search for asymptomatic deficiencies (Clark, 1971). In view of the frequency of duodenal diverticulosis in an elderly population (Pimparkar, 1966), it was felt important to define more precisely their association with deficiency states. A retrospective survey of patients aged $>65$ years and found to have duodenal diverticula at the time of barium meal examinations, during one year in the Cambridge Health District, was therefore carried out.

\section{Methods}

The barium studies were performed in 2 centres. Addenbrooke's Hospital serves as the District General Hospital for the Cambridge Health District and Chesterton Hospital is the Assessment Unit for the Geriatric Department of the same Health District.

All the barium meal examinations in which duodenal diverticula were reported were reviewed, and cases where this could not be retrospectively substantiated were rejected. All diverticula of the first part of the duodenum were rejected as probably representing pseudodiverticula (Pimparkar, 1966).

The case notes of the 39 patients demonstrated to have true duodenal diverticula were reviewed for haematological and biochemical evidence of nutritional deficiency.

\section{Results}

Incidence and age distribution

A total of 451 barium meals were performed in patients aged $>65$ years in the year studied. The mean age in the geriatric hospital was higher $(80.1$ v. 73.2 years) but the proportion of subjects discovered to have duodenal diverticula in the geriatric hospital was much greater (14 of 67 $(20.1 \%) v .25$ of $384(6.5 \%))$.

\section{Symptoms}

The symptoms presented at the time of the investigation are shown in Fig. 1 and are similar to most previous series, (Pimparkar, 1966) but significantly different from that of Clark (1971).

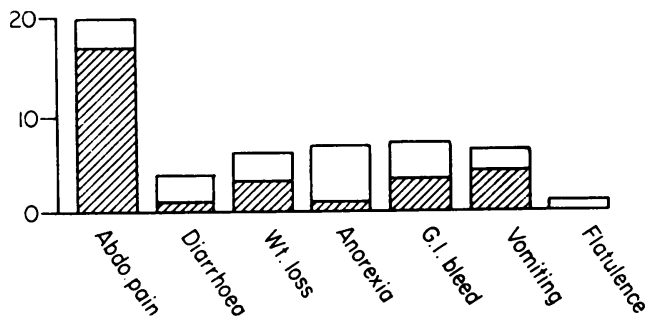

Fig. 1. Duodenal diverticula: symptoms at presentation $(n=51)$. $\square$ All symptoms; $\mathbb{Z}$ principal symptoms.

Ten patients had no symptoms related to the upper gastrointestinal tract and the investigation was performed to elucidate the cause of iron deficiency anaemia (7 subjects) or in an attempt to 
locate a primary neoplasm in patients with known malignant disease ( 3 subjects). The vast majority of these symptoms could be attributed to associated pathology (Table 1).

TABLE 1. Pathology associated with all duodenal diverticula $(n=38)$

\begin{tabular}{lc}
\hline \multicolumn{1}{c}{ Pathology } \\
\hline Hiatus hernia & 10 \\
Duodenal ulcer & 9 \\
Gastric ulcer & 1 \\
Metastatic cancer & 3 \\
Stool occult bleeding alone & 2 \\
Oesophageal ulceration & 1 \\
Alcoholic hepatitis & 1 \\
& -27 \\
\hline
\end{tabular}

Site

The distribution of diverticula was similar to previously reported series (Pimparkar, 1966). Most occurred in the second part of the duodenum with progressively fewer distally.

\section{Haemoglobin}

A low haemoglobin $(<11.5 \mathrm{~g} / \mathrm{dl})$ was discovered in 14 of the 34 subjects tested, but associated pathology (Table 1) to account for this was found in all but one. Since this patient lived alone and had marked intellectual failure, a dietary deficiency seems highly probable.

\section{Serum alkaline phosphatase}

The serum alkaline phosphatase (SAP) had been estimated in 30 patients. A markedly elevated SAP ( $>200$ i.u./l) was found in 5 cases and explicable in all. In only one case was this due to osteomalacia (Case report). SAP values between 90 i.u./l and 140 i.u./l have been discovered to be so common in the elderly that investigation is unrewarding and levels of SAP in this range are regarded as normal.

\section{Serum iron}

Serum iron and total binding capacity (TIBC) were measured in 10 subjects and were normal in only one. In those in whom serum iron was depressed it was uniformly below $7 \mu \mathrm{g} / \mathrm{dl}$. In only one case was a low value inexplicable on the basis of other pathology. This patient probably had a dietary deficiency as described above.

\section{Vitamin $B_{12}$ and folate}

Serum vitamin $B_{12}$ and serum folate and red cell folate levels were measured in 6 patients. One had a low serum folate (Case report) and another had a low serum vitamin $\mathbf{B}_{12}$ level which was subsequently shown to be due to pernicious anaemia.
Mean corpuscular volume (MCV)

In view of the relatively small numbers of subjects in whom vitamin $B_{12}$ and folate and serum iron had been assessed, the distribution of MCV was examined. This had been measured in 32 subjects and was low in $3\left(<76 \mu^{3}\right)$. All these had positive faecal occult blood.

In 6 cases the MCV was elevated, and in 3 cases this could be explained on the basis of known pathology (alcoholic hepatitis metastases, pernicious anaemia). Another had folate deficiency and the remaining 2 were unexplained but had normal serum vitamin $B_{12}$ and folate levels.

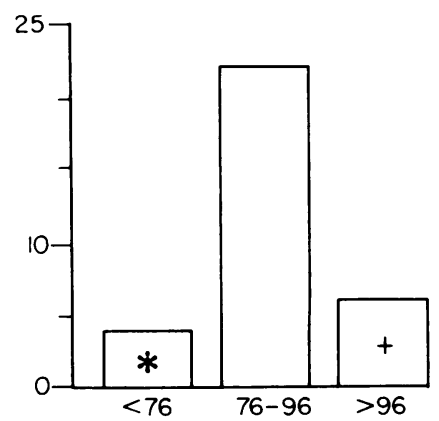

Fig. 2. Mean corpuscular volume $\left(\mu^{3}\right)$. * Positive faecal occu blood: $3 ;+$ alcoholic hepatitis: $1 ;$ metastases: 1 pernicious anaemia: 1 .

\section{Case report}

An 89-year-old female presented with confusion and immobility of recent onset. Other problems noted on admission were deafness, ischaemic heart disease, rheumatoid arthritis, atrophic vaginitis, a lower respiratory infection and an undisplaced fractured neck of femur.

\section{Investigations}

$\mathrm{Hb}, 9.0 \mathrm{~g} / \mathrm{dl} ; \mathrm{MCV}, 97 \mu^{3}$; serum folate $1.8 \mu \mathrm{g} / \mathrm{l}$

(NR 3-18); red cell folate $56 \mu \mathrm{g} / \mathrm{l}(\mathrm{NR}>150)$; faecal occult blood positive on 3 occasions; serum alkaline phosphatase, 1000 (NR 30-92) i.u./1 (heat stability test showed this to be mainly of bony origin); plasma calcium, $1.83 \mathrm{mmol} / \mathrm{l}$ (NR 2-2.6); serum albumin, $31 \mathrm{~g} / \mathrm{l}$; serum iron, $1.4 \mu \mathrm{mol} / 1$, TIBC $9.1 \mu \mathrm{mol} / \mathrm{l}$; barium meal: gastric ulcer and solitary duodenal diverticulum of 3rd part of the duodenum; skeletal survey: multiple rib fractures and Looser's zones in pelvis and femora.

She was placed on folic acid $5 \mathrm{mg}$ twice daily; calcium and vitamin D tablets BP.C, one twice daily; cimetidine, $200 \mathrm{mg}$ thrice daily and $400 \mathrm{mg}$ at night, and oral iron. 
She made a full haematological remission and the alkaline phosphatase fell progressively. The pseudofractures have healed.

One year later she remains haematologically normal and the alkaline phosphatase is in normal limits.

A bile salt breath test using ${ }^{14} \mathrm{C}$-labelled deoxycholic acid was subsequently performed and showed a grossly abnormal result supporting small intestinal bacterial overgrowth.

\section{Discussion}

Deficiency states and malabsorption occur in jejunal diverticulosis and are recognized to be due to contamination of the small intestine (Badenoch, 1958).

Cooke et al. (1963) found the most common abnormality to be vitamin $B_{12}$ deficiency, folate deficiency being less common. In their series only one patient had a low serum iron. Clark (1971) described 15 unselected patients with duodenal diverticula and found a high incidence of nutritional deficiencies of which a low serum iron was most frequent. He suggested that this was related to the level of bacterial contamination in the bowel. However, 2 of the patients described had co-existing jejunal diverticula, one had carcinoid syndrome and one suffered from dermatitis herpetiformis. The latter 2 conditions are now recognized as being associated with malabsorption.

Gillespy (1957) described a case of megaloblastic anaemia and large duodenal diverticulum, but neither vitamin $\mathbf{B}_{12}$ nor folate estimations were recorded. Brey et al. (1971) reported 2 similar cases, but a small bowel biopsy was not performed despite abnormal xylose excretion tests. Goldstein et al., (1963) reported one case with steatorrhoea and a solitary duodenal diverticulum where bacterial contamination was demonstrated and jejunal mucosal architecture was shown to be normal. This seems to be the only reported case where other causes of malabsorption have been excluded. There seems little doubt that bacterial overgrowth may occur in the presence of solitary duodenal diverticula (Gorbach and Tabaqchali, 1969; Tabaqchali and Booth, 1970; Tabaqchali, Hatzioannou and Booth, 1968) and that steatorrhoea and nutritional deficiency may occur in the presence of duodenal diverticula (Brey et al., 1971; Clark, 1971; Gillespy, 1957; Goldstein et al., 1963). That the malabsorption and the diverticula are causally related, however, seems seldom to have been demonstrated. Both are common in the elderly and a true association is yet to be shown in the majority.

One difficulty in interpreting the significance of evidence of bacterial overgrowth is our almost complete ignorance of the normal bowel flora in the very elderly. Although there is only a loose association between malabsorption, small bowel contamination and excess urinary indican excretion (Hamilton et al., 1970), the author has found that a screening test for excess indican in urine was positive in almost half of a group of very elderly patients (unpublished data).

There are thus considerable hazards in comparing the bacterial flora of abnormal elderly people with that of healthy young volunteers. It would not be surprising if the high incidence of small bowel diverticula combined with reduced mobility and frequent hypochlorhydria in elderly subjects made bacterial overgrowth in the small intestine far more frequent than is often assumed.

In the present series of 39 diverticula, only one case of nutritional deficiency seems likely to be related to this lesion, and simple replacement therapy in conventional dosage has corrected this. A bile salt breath test showed strong evidence of small bowel contamination, but neither a dietary deficiency nor gluten-sensitive enteropathy was excluded. The relatively much higher incidence of duodenal diverticula reported in the geriatric unit seems unlikely to be explained on the basis of the slightly higher age range, and is probably related to the effect of interest in those lesions noted by Pimparker (1966). Although the numbers are small the incidence found over a one-year period in the geriatric unit is approximately that which has been reported from careful post-mortem studies employing plaster casts of the duodenum, which is recognized as the most sensitive method of detection (Pimparker, 1966).

The high incidence of deficiencies noted in Clark's (1971) series in the elderly seems likely to have been caused by the inclusion of other disorders in addition to duodenal diverticula, but also by selection in radiological reporting of only those diverticula which were multiple or unusually large.

\section{Conclusions}

Nutritional deficiencies are rarely caused by duodenal diverticula. The importance of these lesions has been exaggerated by isolated reports in which other causes of malabsorption are seldom excluded. Reports of series in which unintentional selection of cases, by radiological reporting which is biased by the interest of the investigator and selective reporting of large or multiple diverticula, has supported this tendency.

In the presence of anaemia or other deficiency states, other causes must actively be sought before concluding that a duodenal diverticulum is responsible. 


\section{References}

BADENOCH, J. (1958) The blind loop syndrome In: Modern Trends in Gastroenterology, 2nd series, (Ed by Jones, F.A.), p. 231. Hüber, New York.

Brey, O.A., Garner, E.P.R., Twycross, R.G. \& Wells, O. (1971) Duodenal diverticulosis and chronic diarrhoea. British Medical Journal, 1, 211.

Clark, A.N.G. (1971) Deficiency states in duodenal diverticular disease. Age and Ageing, 1, 14.

Cooke, W.T., CoX, E.V., Fone, D.J., Meynell, M.J. \& GADDIE, R. (1963) The clinical and metabolic significance of jejunal diverticula. Gut, 4, 115.

GilleSPY, R.O. (1957) Megaloblastic anaemia caused by a posterior duodenal pouch. British Journal of Clinical Practitioners, 11, 48.

Goldstein, F., Cozzolina, H.J. \& WiRTs, W. (1963) Diarrhea and steatorrhea due to a large solitary duodenal diverticulum. American Journal of Digestive Diseases, 8, 937.

GoRbaCh, S.L. \& TABaQchali, S. (1969) Bacterial bile and the small bowel. Gut, 10, 936.

Hamilton, J.D., Dyer, N.H., Dawson, A.M., O'Grady, F.W., Vince, A., Fenton, J.C.B. \& Mollin, D.L. (1970) Assessment and significance of bacterial overgrowth in the small bowel. Quarterly Journal of Medicine, 154, 265.

Pimparkar, B.D. (1966) In: Gastroenterology, (Ed by Bockus, H.L.), Chap. 47. W.B. Saunders, Philadelphia.

TABaQChali, S. \& Booth, C.C. (1970) Modern Trends in Gastroenterology, Vol. 4, (Ed by Card, W.I. \& Creamer, B.), p. 143. Butterworth, London.

Tabaqchali, S., Hatzioannou, J. \& Booth, C.C. (1968) Bile salt deconjugation and steatorrhoea in patients with blind loop syndrome. Lancet, ii, 12. 